FACTA UNIVERSITATIS

Series: Mechanical Engineering Vol. 14, N ${ }^{\mathrm{o}}$ 1, 2016, pp. 21 - 36

Original scientific paper

\title{
MODAL TRIGGERED NONLINEARITIES FOR DAMAGE LOCALIZATION IN THIN WALLED FRC STRUCTURES - A NUMERICAL STUDY
}

\author{
UDC 539.4
}

\begin{abstract}
Tobias Rademacher, Manfred Zehn
Berlin Institute of Technology (TU Berlin), Department of Structural and Computational Mechanics, Germany
\end{abstract}

\begin{abstract}
This paper presents a novel method for detecting locations of damages in thin walled structural components made of fiber reinforced composites (FRC). Therefore, the change of harmonic distortion, which is found by current research to be very sensitive to delamination, under resonant excitation will be derived from FEMsimulation. Based on the linear modal description of the undamaged structure and the damage-induced nonlinearities represented by a nonlinear measure, two spatial damage indexes have been formulated.

The main advantage of this novel approach is that the information about the defect is represented mainly by changes in the modal harmonic distortion (MHD), which just needs to be measured in one (or few) structural points. The spatial resolution is given by the pairwise coupling of the MHD with the corresponding mode shapes.
\end{abstract}

Key Words: Structural Health Monitoring, Composites, Damage Location, Nonlinear Acoustics, Harmonic Distortion, Nonlinear Vibration

\section{INTRODUCTION}

Due to their high stiffness and strength combined with design versatility, FRCs have massively gained in importance in recent years. However, as a consequence of their heterogeneous structure, the thin-walled structural components made of FRC are particularly susceptible to damages in the laminate, which are in most cases characterized by separation of the laminate layers (delamination). These local delaminations can already arise in the manufacturing process. Furthermore, they can be a consequence of a low and moderate energy input, such as bird strikes on aircraft or carelessness in the maintenance.

Received February 1, 2016 / Accepted March 16, 2016

Corresponding author: Tobias Rademacher

TU Berlin, Fachgebiet für Strukturmechanik und -berechnung, Str. des 17. Juni 135, 10623 Berlin, Germany

E-mail: tobias.kaempf@tu-berlin.de 
The particularly critical aspect of this type of damage is the fact that it is visually difficult to detect, spreads as a result of operating loads, and can give rise to total failure.

Therefore, for safety-related applications, it is of crucial importance to be able to detect, locate and assess the defects and damages caused by imperfections in production and/or that appears in operation. The ultrasound and x-ray based inspections are conservative and highly accurate methods. However, they are also quite demanding in terms of the equipment, time and accessibility of the examined structural components. Therefore, especially for larger structures, the methods that are relatively easy to implement are needed in order to enable a coarse localization of potential damages.

In this context, a great number of methods for Structural Health Monitoring (SHM) have been published in the past decades (e.g. reviews in [1-3]). Many of the developed methods are based on a damage-related alteration in the dynamic structural behavior [4, 5]. These methods have also been applied to the structures made of fiber-reinforced composites [6] and extensively studied [7, 8].

\subsection{Linear methods}

The idea that a local damage affects the global vibration response has led to a series of modal-based approaches to structural health monitoring [9]. Most of these "classical" methods are based on the fact that the damage locally reduces the stiffness, thus affecting the natural frequencies [10] and mode shapes, including derived quantities (e.g. modal strain) [11].

A simple method of damage identification has been proposed by Montalvão, Ribeiro and Duarte-Silva [12]. They have extended the principle of damage indicators based on eigenmodes - in this case defined as the Mode-Shape-Curvatures - by weighting the eigenmodes with a relative change in the modal damping, $\eta_{r}$, induced by the damage. They invented the Damping-Damage-Indicator $\left(D a D I_{i}\right)$ which can be obtained by superposition of strain based modal shape functions weighted by $\Delta \eta_{r}$. Montalvão, Ribeiro and Duarte-Silva could prove that it is possible to locate a defect in the composite panel made of carbon fibre prepreg by using the Damping-Damage-Indicator. However, experience has shown that especially the modal damping coefficients in FRC structures strongly depend on temperature. Additionally, the suitability of modal damping as a damage indicator is limited by the diversity of physical causes of damping and the interaction between the structure and the environment, which influences the damping in a way that is hard to forecast and/or control [13].

\subsection{Nonlinear methods}

Methods of nonlinear analysis of the dynamic system response open up many possibilities for structural health monitoring. However, they are often strongly related to a specific problem [14]. The analysis of higher harmonic components of the vibration response in the frequency domain provides a relatively practical approach to damage detection and identification, which is sometimes superior compared to the classical linear methods. Considering a damaged beam, Prime and Shevitz [15] demonstrated that the higher harmonic components in the spectrum and the associated mode shapes are much more sensitive to damages than the modal parameters. 
Considering thin-walled structural components made of FRC, delamination is the most frequent type of damage. Therefore, in the context of structural health monitoring, the research is focused on its impact on the dynamic behavior [16]. Experimental investigations carried out on a delaminated beam show that the alternating opening and closing of a delamination gap (delamination breathing) particularly changes the vibrational response compared to the undamaged case [17-19]. The beating normal contact between the gap's opposite surfaces and the resulting direction-dependent stiffness leads to a non-linear structural behavior as a consequence of an abrupt state change. Hence, when such a system is exposed to a harmonic excitation, the response spectrum is characterized by the occurrence of higher harmonics, obtained as integer multiples of the excitation frequencies [20, 21]. These nonlinearities can be quantified, for instance, in an integral form by means of the clapping factor [22].

\section{THE IDEA OF NONLINEAR DAMAGE INDEX}

The new method presented in this paper aims at the coarse localization of damages within thin-walled structural FRC components made of fiber-reinforced composites in order to get an overview for more detailed investigations. For this purpose, usually weak non-linear effects in the vibration response, which are the consequence of the damage, should be quantified by means of a suitable quantity $\lambda_{r}$. In the present work this will be a value derived from harmonic distortion.

The basic idea is that the structure is exposed to a resonant vibration excitation. It is also necessary that, despite of the damage, the original modal representation can still sufficiently describe the overall dynamic behavior of the structure, which implies that the damage induced changes in eigenmodes and eigenfrequencies are still negligible. The validity of this assumption has been confirmed in the literature (for example in $[18,23$, 24]) in the case of slighter damages of the laminates. Hence, a damage-induced nonlinear measure $\lambda_{r}$ is assigned to the $r^{\text {th }}$ mode (see Fig. 1).

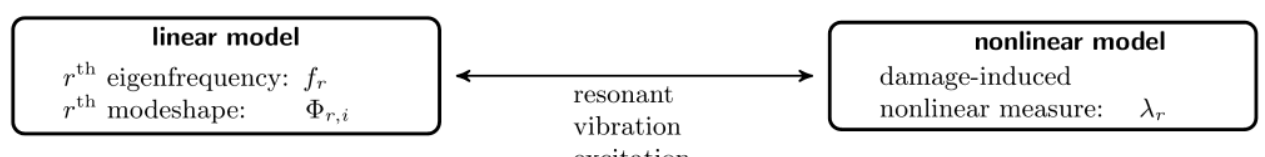

Fig. 1 Coupling of nonlinear-measure, modal strain and damage

Using a suitable combination of linear modal description of the original structure and nonlinear measure $\lambda_{r}$ associated with damage-induced changes, a spatial damage index, nonlinear damage index $N L D I_{i}$, could be formulated in a similar way as was done by Montalvão, Ribeiro and Duarte Silva [12]:

$$
N L D I_{i}:=\frac{\sum_{r=1}^{N} \Delta \lambda_{r} \tilde{\Phi}_{r, i}}{\sum_{r=1}^{N} \tilde{\Phi}_{r, i}}, \quad \text { where here } \quad \tilde{\Phi}_{r, i}:=\frac{\left|\bar{\kappa}_{I, i}+\bar{\kappa}_{I I, i}\right|}{\max \left|\bar{\kappa}_{I,:}+\bar{\kappa}_{I I,:}\right|} .
$$




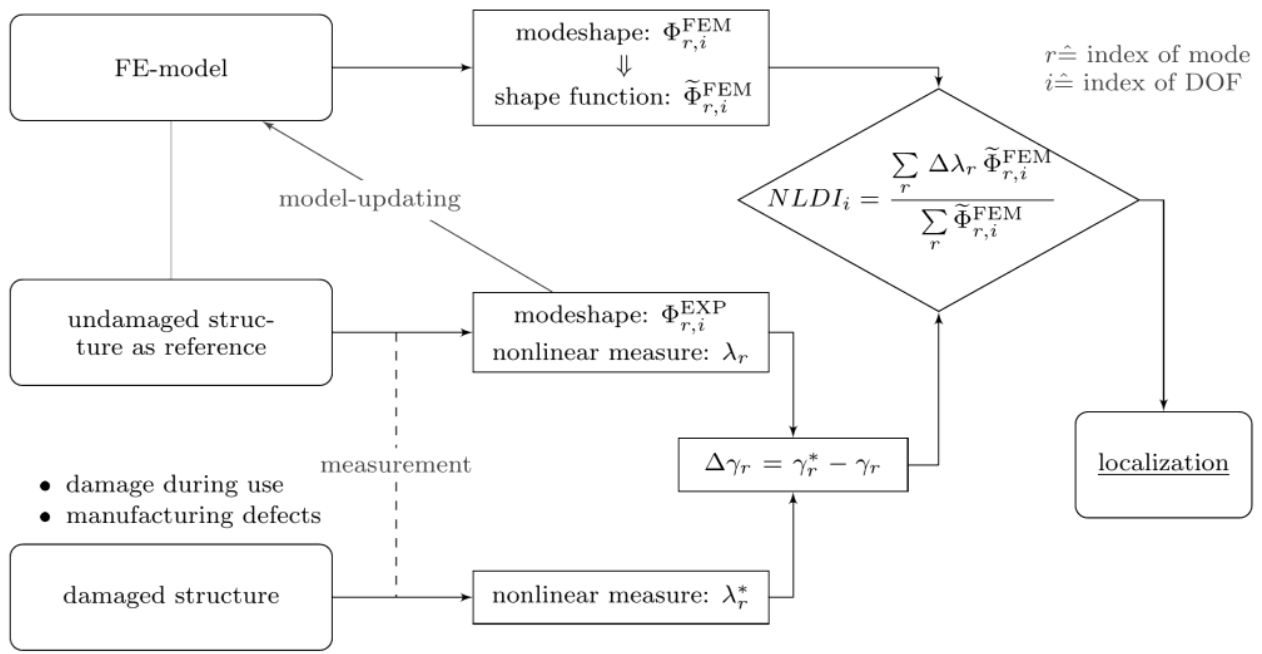

Fig. 2 Flow chart of the suggested damage localization approach

The necessary steps of the proposed method are depicted in Fig. 2. A prerequisite for successful damage localization is a valid linear FE model of the structure not including damages. This enables the determination of optimal measurement and excitation points for the experimental determination of $\lambda_{r}$. On the other hand, each eigenmode or a derived quantity, e.g., the curvature-dependent shape function used here as defined in Eq. (1), can be represented with a high spatial resolution.

Nonlinear measures $\lambda_{r}$ associated to the modes, are obtained by means of comparative measurements carried out on the original and potentially damaged structure. Change $\Delta \lambda_{r}$, is supposed to serve not only as a damage indicator, but also for the localization by means of proposed damage index NLDI. The formulation given in Eq. (1) takes advantage of an increased distortion when the referred mode shape is triggering the local damage induced nonlinear mechanism. Likewise, the linearity of each mode can be used to define a reciprocal nonlinear damage index:

$$
r N L D I_{i}:=\left(\frac{\sum_{r=1}^{N} \frac{1}{\Delta \lambda_{r}} \tilde{\Phi}_{r, i}}{\sum_{r=1}^{N} \tilde{\Phi}_{r, i}}\right)^{-1} .
$$

\section{NUMERICAL PROOF OF THE CONCEPT}

This section describes how the nonlinear response is obtained for test purposes by a numerical simulation of an experimental set up. All calculations stated below have been done using MATLAB. 


\subsection{Finite element model of a thin fiber reinforced composite plate}

For the numerical proof of the concept a rectangular glass fiber-reinforced polymer plate (GFRP) is modeled using the finite element method. For the sake of convenience it is assumed that the balanced and symmetric cross ply $\left(0^{\circ} / 90^{\circ}\right)$ laminate is used. Thus, by applying the classical laminate theory (see [25]) the membrane and bending problem is decoupled. Because of being easily stimulated and measured in the experiment, only bending modes will be considered here. Using the first order shear deformation theory the displacement field can be written similarly to the Reissner-Mindlin plate model as:

$$
\begin{aligned}
\mathbf{u}(x, y, z)=\left[\begin{array}{lll}
u & v & w
\end{array}\right]^{T} & =\left[\begin{array}{lll}
-z \frac{\partial w}{\partial x} & -z \frac{\partial w}{\partial y} & w
\end{array}\right]^{T} \\
& =\left[\begin{array}{llll}
z \vartheta_{y}(x, y) & -z \vartheta_{x}(x, y) & w(x, y)
\end{array}\right]^{T}
\end{aligned}
$$

By applying derivation to the displacements the strain field becomes:

$$
\boldsymbol{\kappa}=\left[\begin{array}{lll}
\kappa_{x x} & \kappa_{y y} & \kappa_{x y}
\end{array}\right]^{T}=\left[\begin{array}{lll}
-\frac{\partial \vartheta_{y}}{\partial x} & \frac{\partial \vartheta_{x}}{\partial y} & \frac{\partial \vartheta_{x}}{\partial x}-\frac{\partial \vartheta_{y}}{\partial y}
\end{array}\right]^{T}
$$

and

$$
\boldsymbol{\gamma}=\left[\begin{array}{ll}
\gamma_{x z} & \gamma_{y z}
\end{array}\right]^{T}=\left[\begin{array}{ll}
\frac{\partial w}{\partial x}+\vartheta_{y} & \frac{\partial w}{\partial y}-\vartheta_{x}
\end{array}\right]^{T}
$$

where the vectors for curvatures $\mathbf{\kappa}$, resulting from bending moments $\mathbf{m}$, and transverse shear strain $\boldsymbol{\gamma}$, due to shear forces $\mathbf{f}_{s}$, can be written separately.

The equation of motion can be derived by using Hamilton's principle:

$$
\delta \int_{t_{1}}^{t_{2}}\left\{T\left(p_{i}, \dot{p}_{i}\right)-\Pi\left(p_{i}\right)\right\} d t-\int_{t_{1}}^{t_{2}} \delta^{\prime} W d t=0 \text { with } \delta p_{i}\left(t_{1}\right)=\delta p_{i}\left(t_{2}\right)=0 \quad \forall i
$$

The generalized coordinates identified as independent "displacements" are:

$$
\mathbf{p}=\left[\begin{array}{lll}
p_{1} & p_{2} & p_{3}
\end{array}\right] \equiv\left[\begin{array}{lll}
w & \vartheta_{x} & \vartheta_{y}
\end{array}\right] \text {. }
$$

Including the assumptions above, kinetic energy $T$ follows to:

$$
T=\frac{1}{2} \int_{V} \rho \dot{u}^{2} \quad d V=\frac{1}{2} \int_{A} t \rho \dot{w}^{2} d A+\frac{1}{2} \int_{A} \frac{t^{3}}{12} \rho\left(\dot{\vartheta}_{x}^{2}+\dot{\vartheta}_{y}^{2}\right) d A
$$

Applying the classical laminate theory (see [25]) and calculating the shear correction factors [26], strain energy $\Pi$ can be integrated over the thickness:

$$
\Pi=\frac{1}{2} \int_{V}\left(\mathbf{m}^{T} \mathbf{\kappa}+\mathbf{f}_{s}^{T} \boldsymbol{\gamma}\right) \quad \mathrm{d} V \stackrel{C L T}{=} \frac{1}{2} \int_{A}\left(\boldsymbol{\kappa}^{T} \mathbf{D} \mathbf{\kappa}+\boldsymbol{\gamma}^{T} \mathbf{S} \boldsymbol{\gamma}\right) \mathrm{d} A
$$


where with the above given assumptions the laminate bending and shear stiffness matrices have the form:

$$
\mathbf{D}=\left[\begin{array}{ccc}
D_{11} & D_{12} & 0 \\
& D_{22} & 0 \\
\text { sym. } & & D_{66}
\end{array}\right] \text { and } \mathbf{S}=\left[\begin{array}{cc}
S_{11} & 0 \\
0 & S_{22}
\end{array}\right] \stackrel{\text { here }}{=}\left[\begin{array}{cc}
S_{11} & 0 \\
0 & S_{11}
\end{array}\right] \text {. }
$$

Virtual work $\delta$ ' $W$ of non-conservative and external loads acting on plates surface $A$ and its Edges $s$ becomes:

$$
\delta W=\int_{V} \mathbf{R} \delta \mathbf{p} \mathrm{d} V+\int_{A} \mathbf{P} \delta \mathbf{p} \mathrm{d} A+\int_{s} \mathbf{F} \delta \mathbf{p} \mathrm{d} s
$$

For discretization of plate surface $A$ a planar four-node $(N e=4)$ quadrilateral element with bilinear shape functions $N_{i}$ is used. Thus, element solution $\mathbf{u}^{e}$ of the displacement model for the plate is expressed as:

$$
\mathbf{u}^{e} \doteq \mathbf{N} \mathbf{q}^{e}=\sum_{i=1}^{N e} \mathbf{N}_{i} \mathbf{q}_{i}=\sum_{i=1}^{N e}\left[\begin{array}{ccc}
N_{i} & 0 & 0 \\
0 & 0 & N_{i} \\
0 & N_{i} & 0
\end{array}\right]\left[\begin{array}{c}
\mathrm{w}_{i} \\
\vartheta_{x i} \\
\vartheta_{y i}
\end{array}\right] .
$$

With substituting the displacement fields in Eq. (6) with these interpolation functions for one element domain the dynamical equilibrium reads:

$$
\int_{t_{l}}^{t_{2}}\left\{\int_{A_{e}} \mathbf{N}^{\mathbf{T}} \tilde{\mathbf{M}} \mathbf{N} \mathrm{d} A \ddot{\mathbf{q}}^{e}+\mathbf{C}^{\mathrm{e}} \dot{\mathbf{q}}^{\mathrm{e}}+\int_{A_{e}} \mathbf{B}_{b}^{T} \mathbf{D} \mathbf{B}_{b}+\overline{\mathbf{B}}_{s}^{T} \mathbf{S} \overline{\mathbf{B}}_{s} \mathrm{~d} A \mathbf{q}^{e}-\mathbf{f}_{e x t}^{e}-\mathbf{f}_{i n t}^{e}\right\} \delta \mathbf{q}^{e} \mathrm{~d} t=0,
$$

where dissipative loads are assumed to be proportional to the nodes velocity.

Because times $t_{1}$ and $t_{2}$ and virtual node displacements $\delta \mathbf{q}^{e}$ can be chosen arbitrarily this finally yields to:

$$
\begin{gathered}
\int_{A_{e}} \mathbf{N}^{\mathbf{T}} \tilde{\mathbf{M}} \mathbf{N} \mathrm{d} A \ddot{\mathbf{q}}^{e}+\mathbf{C}^{e} \dot{\mathbf{q}}^{e}+\int_{A_{e}} \mathbf{B}_{b}^{\mathrm{T}} \mathbf{D} \mathbf{B}_{b}+\overline{\mathbf{B}}_{s}^{T} \mathbf{S} \overline{\mathbf{B}}_{s} \mathrm{~d} A \mathbf{q}^{e}=\mathbf{f}_{e x t}^{e}+\mathbf{f}_{i n t}^{e}, \\
\mathbf{M}^{e} \ddot{\mathbf{q}}^{e}+\mathbf{C}^{e} \dot{\mathbf{q}}^{e}+\left(\mathbf{K}_{b}^{e}+\mathbf{K}_{s}^{e}\right) \mathbf{q}^{e}=\mathbf{f}_{e x t}^{e}+\mathbf{f}_{i n t}^{e},
\end{gathered}
$$

where consistent element mass matrix $\mathbf{M}^{e}$ follows from numerical integration of the shape functions with the diagonal Kronecker product

$$
\tilde{\mathbf{M}}:=\rho t \mathbf{I}_{4 \times 4} \otimes\left[\begin{array}{lll}
1 & \frac{1}{12} t^{2} & \frac{1}{12} t^{2}
\end{array}\right]
$$

The bending component of the element stiffness matrix is set up by using straindisplacement matrix $\mathbf{B}_{b}$ according to 


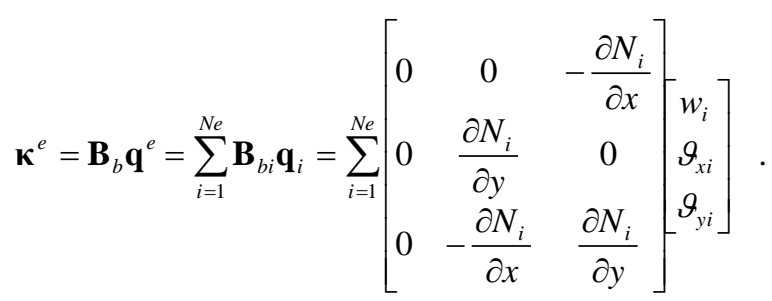

In order to avoid shear locking and hourglass modes (which might cause instabilities in time integration), the shear component of the element stiffness matrix is calculated by using the assumed natural strain approach (see [27]). Therefore, the out-of-plane shear strains at edge centers are firstly calculated. Subsequently, these values are used to interpolate shear strain field $\gamma$ :

$$
\boldsymbol{\gamma}^{e}=\overline{\mathbf{B}}_{s} \mathbf{q}^{e} .
$$

Due to the integral form of Eq. (6) the dynamical equilibrium for the whole plate domain follows with Eq. (15):

$$
\sum_{e=1}^{N E} \mathbf{M}^{e} \ddot{\mathbf{q}} \ddot{e}^{e}+\mathbf{C}^{e} \dot{\mathbf{q}}^{e}+\mathbf{K}_{b}^{e} \mathbf{q}^{e}=\sum_{e=1}^{N E} \mathbf{f}_{e x t}^{e}+\mathbf{f}_{\text {int }}^{e} \Rightarrow \mathbf{M} \ddot{\mathbf{q}}+\mathbf{C} \dot{\mathbf{q}}+\mathbf{K q}=\mathbf{f}_{e x t}
$$

which can be integrated in time for a given external load vector $\mathbf{f}_{e x t}$ and the initial conditions for node displacements $\mathbf{q}$ and velocities $\dot{\mathbf{q}}$ (see section 3.3).

In the present work an unbounded plate with the characteristics as shown in Table 1 was discretized using a regular quadrilateral mesh with $12 \times 24$ or $14 \times 28$ elements. Both configurations show a maximum error for the desired eigenfrequencies of $1 \%$ with respect to ABAQUS model ( $24 \times 48$ quadratic elements type S8R).

In order to suppress rigid body motion and to achieve reasonable damping for the elastic modes a viscoelastic foundation is set up. So the frequencies of significantly damped rigid body modes are smaller than $1 \%$ of the first elastic mode and the modal damping of the elastic modes become approximately $0.1 \%$, which is in good agreement with experimental studies.

Table 1 Characteristics of the glass fiber-reinforced polymer plate

\begin{tabular}{ccccccccc}
\hline Length $a$ & Width $b$ & Thickness $t$ & Density $\rho$ & $D_{11}$ & $D_{22}$ & $D_{12}$ & $D_{66}$ & $S_{11}=S_{22}$ \\
\hline $0.24 \mathrm{~m}$ & $0.48 \mathrm{~m}$ & $3.5 \mathrm{~mm}$ & $2100 \mathrm{~kg} / \mathrm{m}^{3}$ & $71.5 \mathrm{Nm}$ & $98.6 \mathrm{Nm}$ & $8.6 \mathrm{Nm}$ & $13.3 \mathrm{Nm}$ & $99.7 \mathrm{MNm}^{-1}$ \\
\hline
\end{tabular}

\subsection{Modeling the time dependent behavior of delamination}

Despite a great research effort, it is still not very well understood how the physical mechanisms appearing in delaminated structures affect the nonlinearities in dynamic response. For the wavelength range investigated in this paper, the phenomena on macroscopic scale [28], namely the clapping mechanism (see [29, 20]) and bilinear stiffness due to opening and closing the delamination [30], will affect the vibrational response mostly. Modeling the former would require a very fine spatial and time resolution of the delaminated area to represent the governing contact mechanisms. Taking 
into account that for the method proposed in this paper many time integration steps (for every mode) over a quite long period of time (essential to obtain a sufficient frequency resolution) need to be calculated, this phenomenon is therefore not feasible here. Whereas the state-dependent impedance mismatch, caused by a slight local stiffness reduction in the case of an open-state delamination, can be easily modeled with a lumped parameter model [31, 32].

In this paper the local curvature at the damage position is assumed to determine whether the delamination is open or closed and, accordingly, the local stiffness is reduced or not. Therefore, one characteristic value $\bar{\kappa}$, representing an overall curvature based on the local values given in Eq. (17), has to be defined. Therefore, the major curvature calculated by solving the eigenvalue problem:

$$
\begin{gathered}
\operatorname{det}\left[\begin{array}{cc}
\kappa_{x x}-\mu & \kappa_{x y} \\
\kappa_{x y} & \kappa_{y y}-\mu
\end{array}\right] \stackrel{!}{=} 0 \\
\mu_{I / I I} \equiv \kappa_{I / I I}=\frac{1}{2}\left(\kappa_{x x}+\kappa_{y y}\right) \pm \sqrt{\left(\frac{1}{2}\left(\kappa_{x x}-\kappa_{y y}\right)\right)^{2}+\kappa_{x y}^{2}} .
\end{gathered}
$$

The large magnitude is supposed to govern the delamination state (see Fig. 3). Hence, characteristic curvature value $\bar{\kappa}$ is defined by:

$$
\bar{\kappa}:=\kappa_{I}+\kappa_{I I} \rightarrow\left\{\begin{array}{lc}
\bar{\kappa} \leq 0 & \text { delamination closed } \\
\bar{\kappa}>0 & \text { delamination open }
\end{array} .\right.
$$

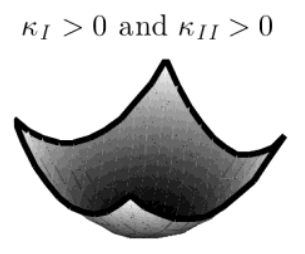

$$
\kappa_{I}<0 \text { and } \kappa_{I I}<0
$$

$$
\kappa_{I}>0 \text { and } \kappa_{I I}<0
$$
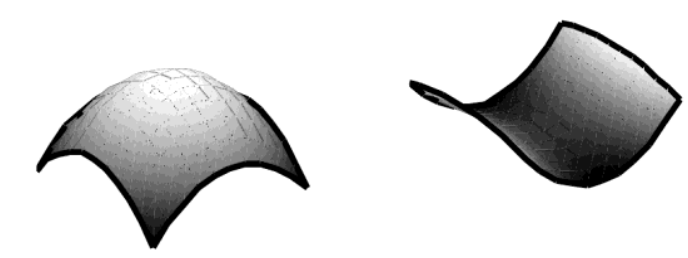

Fig. 3 Major curvatures

All element stiffness matrices $\mathbf{K}^{e^{*}}$ corresponding to delaminated area are assumed to undergo a curvature-dependent degradation like:

$$
\mathbf{K}^{e^{*}}=(1-r(\bar{\kappa})) \mathbf{K} \quad \text { where } r(\bar{\kappa})=\left\{\begin{array}{cc}
0 & \text { for } \bar{\kappa} \leq 0 \\
\hat{r}\left(1-e^{\frac{1}{2\left(\frac{\bar{\kappa}}{\bar{\kappa}}\right)^{2}}}\right. & \text { else. }
\end{array}\right.
$$

where reasonable values for maximum degradation $\hat{r}$ range between 0 (no reduction) and 1 (no remaining element stiffness). As shown in Fig. 4 the course of degradation is a Gaussian smoothed step function where the smoothness is controlled by value $\hat{\kappa}$. This 
smoothed shape is chosen instead of a sharp heavy side function for two reasons: on the one hand, delamination with its natively non-regular topology will not act upon a strict all-or-nothing law. On the other hand, the numerical time integration becomes less critical for discretization errors near the reversal point. This can finally improve the (physical) stability of the integration process.
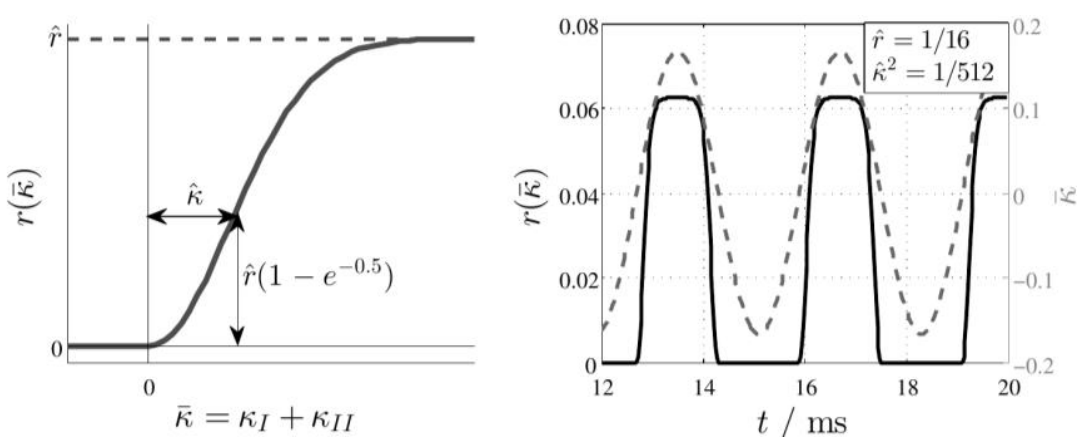

Fig. 4 Curvature dependent stiffness reduction $r$ (left) and its time response

\subsection{Time integration}

To achieve the slightly nonlinear vibrational response of the damaged plate, the discretized equation of motion given in Eq. (15) has to be integrated in time until a steady state (or limit cycle) is reached. To avoid numerical overhead caused by transient oscillation with stimulated eigenfrequency $f_{r}$ the linear steady state solution is set up as the initial condition. To obtain a steady solution of the distorted response 32 periods of main frequency $\left(T_{p r e}=32 / f_{r}\right)$ are processed until the time span $\left(T_{a n a}=32 / f_{r}\right)$ used for spectral analysis starts. To ensure a sufficient resolution of the degradation process one period is sampled with 128 time steps. Hence the sampling data for each mode $r$ follows as:

$$
\Delta t=\frac{1}{f_{s}} \doteq \frac{1}{128} \frac{1}{f_{r}} \text { and } T_{\text {total }}=T_{\text {pre }}+T_{\text {ana }} \doteq(32+32) \frac{1}{f_{r}} .
$$

To provide comparability between the modal triggered nonlinearities the excitation level has to be equalized for all the modes with respect to the related mode shape derivate. Here the curvature is assumed to be the governing characteristic related to generation of nonlinearity. Hence, each modal excitation force $\mathbf{f}_{e x t, r}$ has been tuned in such a manner that for every mode the same rms-value of the characteristic curvature is achieved:

$$
\mathbf{f}_{e x t, r}=\hat{\mathbf{f}}_{e x t, r} \cos \left(2 \pi f_{r} t\right) \quad \text { where } \quad \hat{\mathbf{f}}_{e x t, r} \leftrightarrow \sqrt{\left\langle\bar{\kappa}_{r}^{2}\right\rangle}=\text { const. } \forall r
$$

The time integration is carried out by using the implicit HHT- $\alpha$ method (see [33]). In this generalization of the Newmark- $\beta$ method, the equations of motion are modified, using a parameter $\alpha$, which causes a numerical lag in internal and external forces. This method is chosen for two reasons. On the one hand, using the correct parameters it becomes at least second-order accurate and unconditionally stable. On the other hand, in this way it tends to dampen-out the response at high frequencies which is desirable for physical stability. 


\subsection{Extracting the nonlinear measure from time series}

As described above the nonlinear measure is extracted from a spectrum of a steady state response in one or only a few testing points. Therefore, the last 32 periods of the corresponding velocity signals are analyzed using the Fast Fourier Transform. In order to minimize the amplitude error the flat-top window HFT116D as suggested in [34] is used to process linear power spectra $L P S$.
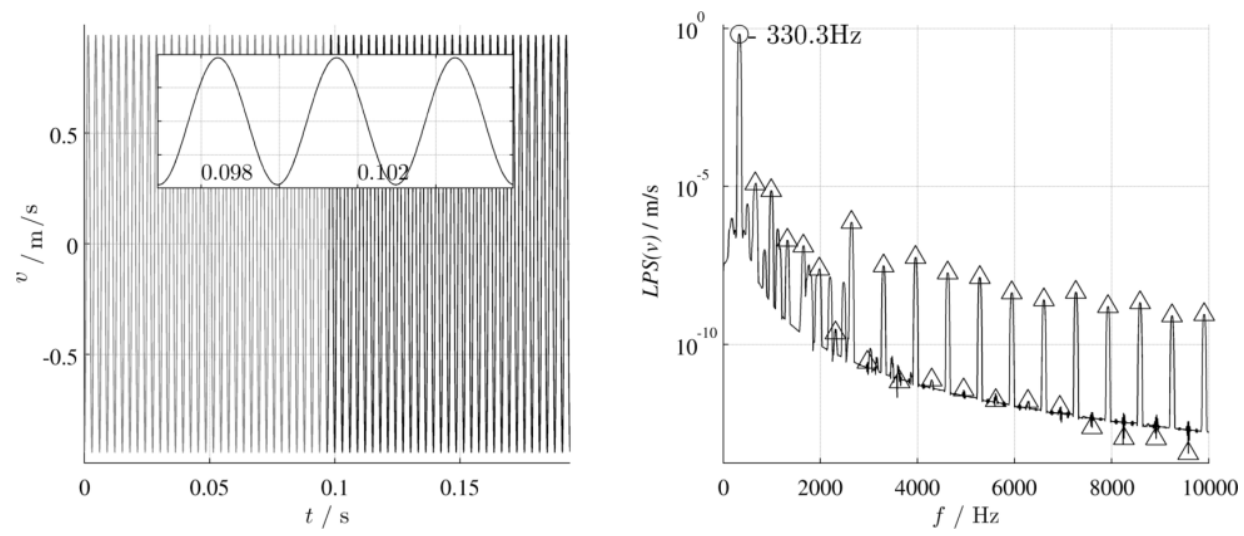

Fig. 5 Typical whole time response of nodes velocity (left) including a detailed view. 64 periods of third mode $(330.3 \mathrm{~Hz})$ are shown, where the last 32 are used to calculate the linear power spectrum (right). The higher harmonics are marked

As shown in Fig. 5 the peaks of higher harmonics $\left(f_{h n}=n f_{r}\right.$ with $\left.n=2,3, \ldots\right)$ of (linear) frequency $f_{r}$ can be clearly determined. Starting with the $7^{\text {th }}$ harmonic a significant alternating behavior - low amplitudes for odd and high for even orders - can be seen. This is the reversal to the pattern which can be observed analyzing a SDOF-system having a bilinear stiffness (see [28]) and might be caused by the more complex modeling of damaged changed stiffness.

In the present work nonlinear measure $\lambda_{r}$, which characterizes the nonlinearity, is estimated by the sum of the first four peaks of higher harmonics:

$$
\lambda_{r}^{i}:=\sum_{n=2}^{5} \operatorname{LPS}\left(\dot{w}_{i}\right)\left[n f_{r}\right] \text { and } \lambda_{r}=\frac{1}{M} \sum_{i=1}^{M} \lambda_{r}^{i}
$$

If more than one measurement point is used, then the overall nonlinear measure is simply averaged mode wise.

Of course, a more complex and maybe more significant estimation rule could be deduced, e.g., discrimination between even and odd higher harmonics. However, as stated below, even this simple way leads to very promising results. 


\section{RESULTS}

In the present work the suggested approach for damage localization has been tested by a numerical simulation of experiments using the assumptions made in section 3 . The first 42 elastic modes of the undamaged structure, ranging between $135.6 \mathrm{~Hz}$ and $6272 \mathrm{~Hz}$, are stimulated and analyzed as described above.

\subsection{Damage index for rectangular FRC-plate with a single damaged area}

A typical distribution of the plain (Eq. (1)) and the reciprocal (Eq. (2)) nonlinear damage indexes is displayed in Fig. 6. It is obvious that both of them show a symmetric pattern, which is a consequence of the fact that underlying shape functions (see Eq. (1)) are symmetric as well.

However, despite this, both the indexes can sufficiently localize the defective area with the use of only one measurement point, where nonlinear measure $\lambda_{r}$ is determined. This result varies only slightly when this measurement point is changed or if more points are taken into account. This is a major advantage of the proposed method: only a few measurement points have to be equipped with sensors while the structure is under test.

While using the plain formulation of the damage index the shape functions seem to be "showing through" and some local maxima become apparent, but the reciprocal form gives a very clear result. This difference can be observed for most of the studied damage positions. But in some cases $r N L D I$ tends to highlight whole lines or columns of elements (compare to Fig. 9). This might be caused by the strong repetitive characteristics of the mostly purely sine-like eigenforms of the plate under investigation.
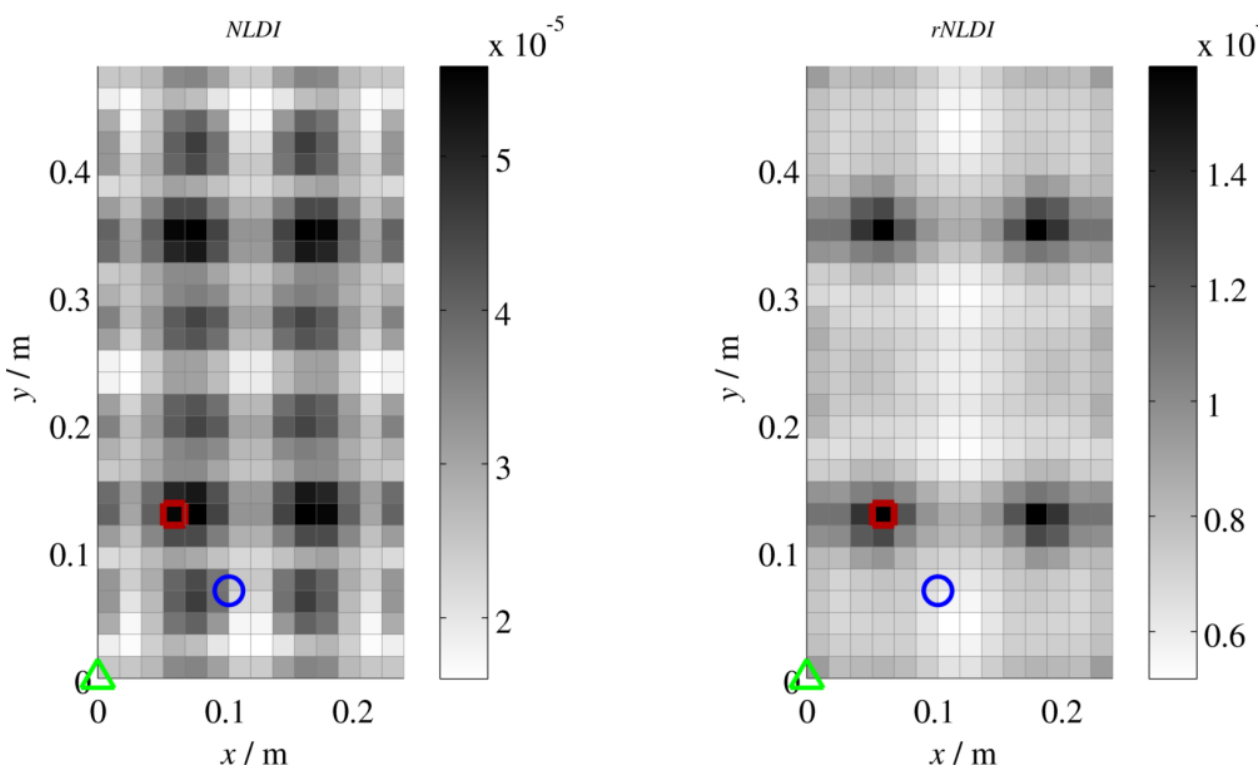

Fig. 6 Linear NLDI (left) and reciprocal $r N L D I$ (right) nonlinear damage index.

Excitation at $(0,0)$ (green) and the nonlinear measures are taken at $(0.1 \mathrm{~m}, 0.07 \mathrm{~m})$ (blue). Only one element $(0.05 \mathrm{~m}, 0.12 \mathrm{~m})$ is damaged (red) 


\subsection{Influence of position and characteristics of the damaged area}

It can be seen in Fig. 7 that the value of modal nonlinear measure $\lambda_{r}$ scales very well with the squared maximum degradation. In case of heavy stiffness reductions $(25 \%$ and higher), significantly distorted modes tend to slightly lower values for scaled $\lambda_{r}$. However, the influence of the maximum degradation to relative pattern of $\lambda_{r}$ is negligible. Hence, it does not affect the validity of the damage index. But it has to be noted that the observed quadratic dependency of $\lambda_{r}$ with respect to degradation might cause resolution problems in experimental studies.

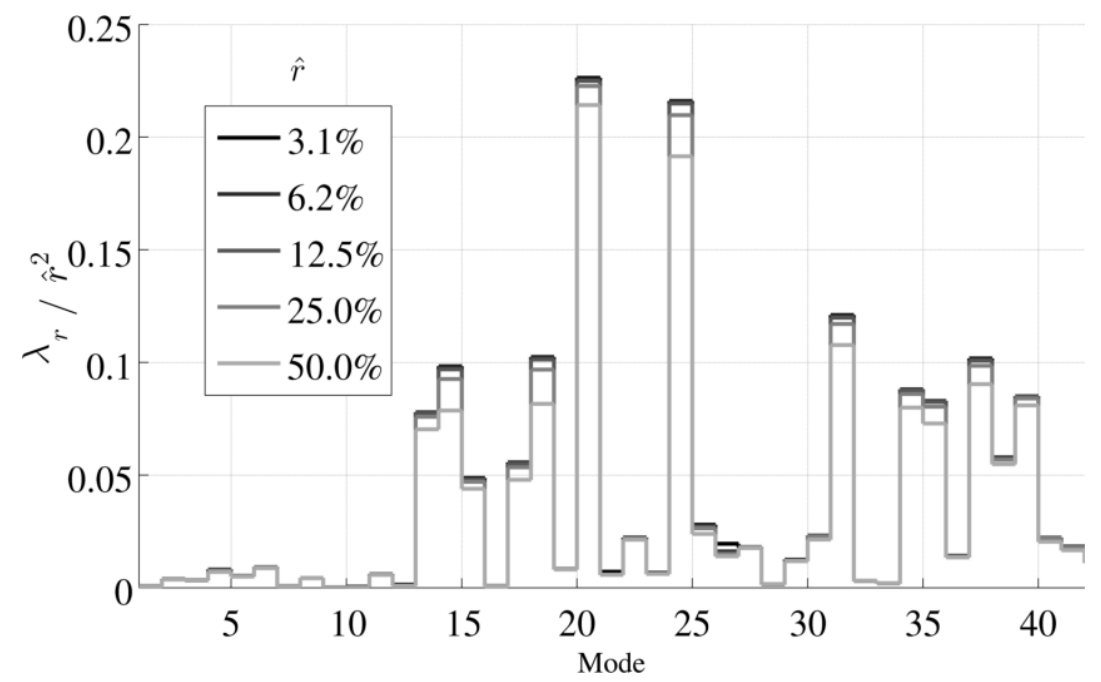

Fig. 7 Scaled nonlinear measures $\lambda_{r} / r^{2}$ for different values of maximum degradation $\hat{r}$. Excitation at $(0,0)$. Nonlinear measures are taken at $(0.02 \mathrm{~m}, 0.04 \mathrm{~m})$. Only one element is damaged

To ensure that a local damage can be found for any location, a huge amount of complete simulation cycles have been carried out. For every single element of the lower left quadrant of the plate ( $x$ in $[0,0.5 a], y$ in $[0,0.5 b]$ ) damage is applied and the complete time integration procedure is performed. Subsequently, the spatial damage indices $N L D I$ and $r N L D I$ have been calculated. In order to decide whether the damage could be uniquely identified, the damage index ratio is computed between the real damage position and the maximum index value of the plate excluding the immediate surroundings of the damage. The result can be seen in Fig. 8.

It can be observed that both the plain and the reciprocal nonlinear damage indexes succeed in the identical (excluding effects of symmetry) location of the damaged area in most cases. The distribution of values and, therefore, the few damage positions in which no identical localization was possible seems to be randomly distributed. However, using both proposed damage indices should ensure a coarse localization of damage for the engineering praxis as a preliminary check for more elaborate local investigations. 

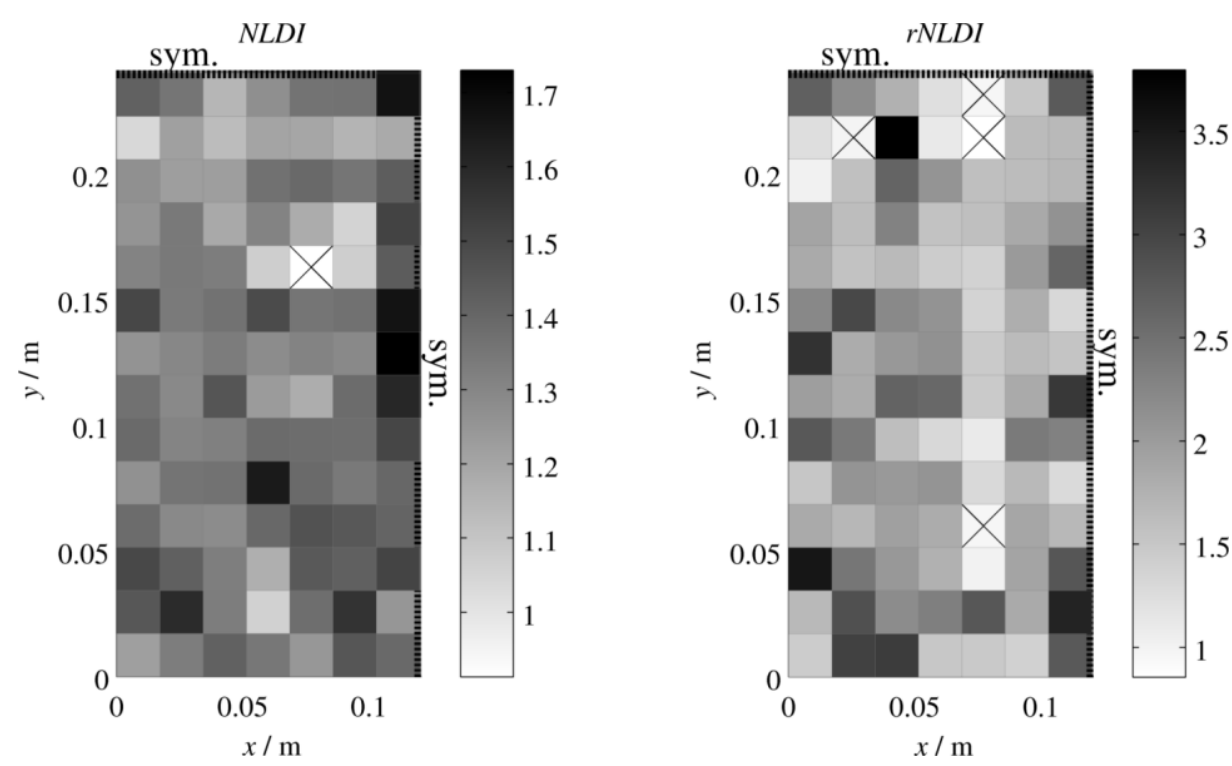

Fig. 8 Uniqueness of the nonlinear damage index and its reciprocal representation for the lower (left) quadrant. Damage positions were the damage index could not uniquely identified (values $<1)$ are marked with a cross. Excitation at $(0,0)$. Nonlinear measures are taken at $(0.02 \mathrm{~m}, 0.04 \mathrm{~m})$

An increase of the spatial spread of the delamination is implemented by involving more damaged elements. It is found that with an increased damaged area the values of the modal nonlinear measure rise as well although the significant differences between them which can be observed for small damages (see Fig. 7) seem to vanish. Hence, as shown in Fig. 9, the significance of the damage index is reduced. While for one damaged element
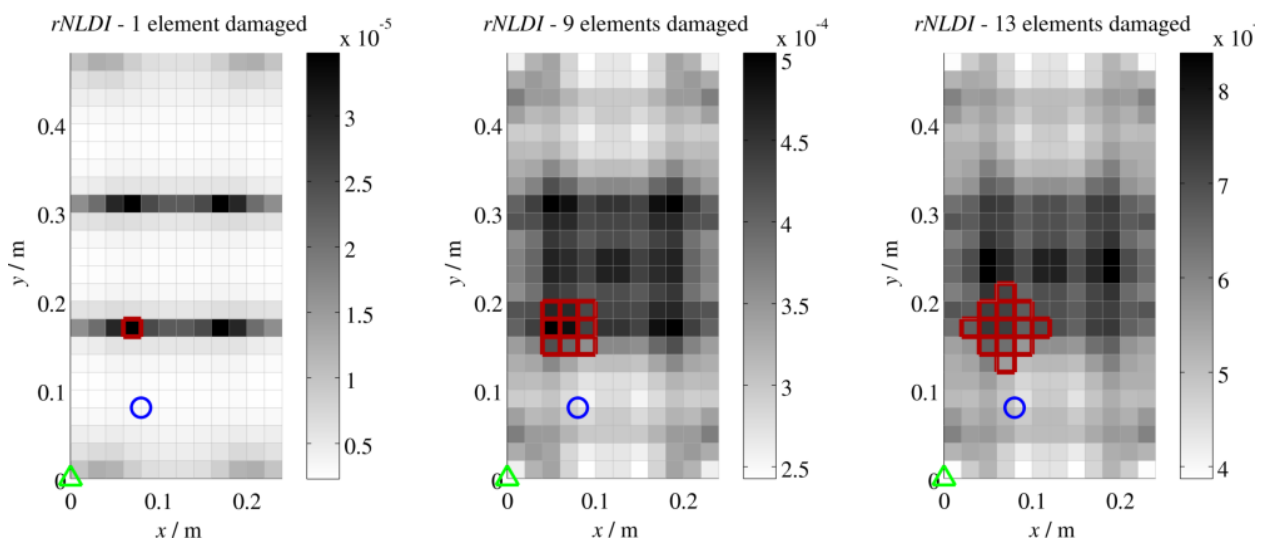

Fig. 9 Reciprocal damage index for different spatial extends of damage. Damaged elements (degradation $\bar{r}=12.5 \%$ ) are highlighted red. Excitation at $(0,0)$ (green). Nonlinear measures $\lambda_{r}$ are taken at $(0.08 \mathrm{~m}, 0.08 \mathrm{~m})$ (blue) 
$r N L D I$, regarding effects of symmetry, matches the damaged position, the affected area in the case of nine damaged elements is overestimated. However, the largest value is still located in the damaged area. In case of 13 damaged elements the supposed method fails completely - which can be slightly improved by using more points to estimate the nonlinear measure. This behavior is inherent for this approach. The larger the damaged area becomes, the more mode shapes are triggering the nonlinearities. So the weights for the superposed shape functions get equalized.

\subsection{Damage Index for a single damaged asymmetric FRC-Structure}

Finally, the proposed method is tested for asymmetric structures, which might be more relevant for real world applications. Even symmetric structures can be easily misbalanced by a slight change in the boundary conditions or by attaching (known) masses while the device is under test.

A simple asymmetric geometry, based on the rectangular plate as described above, can be seen in Fig. 10. It appears that both the indices mark the defective element with the highest value. While the linear damage index shows some areas having falsely high values in the lower part, the reciprocal nonlinear damage index fits perfectly. Once more it has to be emphasized that the nonlinear measure is the only value related to the damaged structure and this is achieved with only one (!) measurement point.
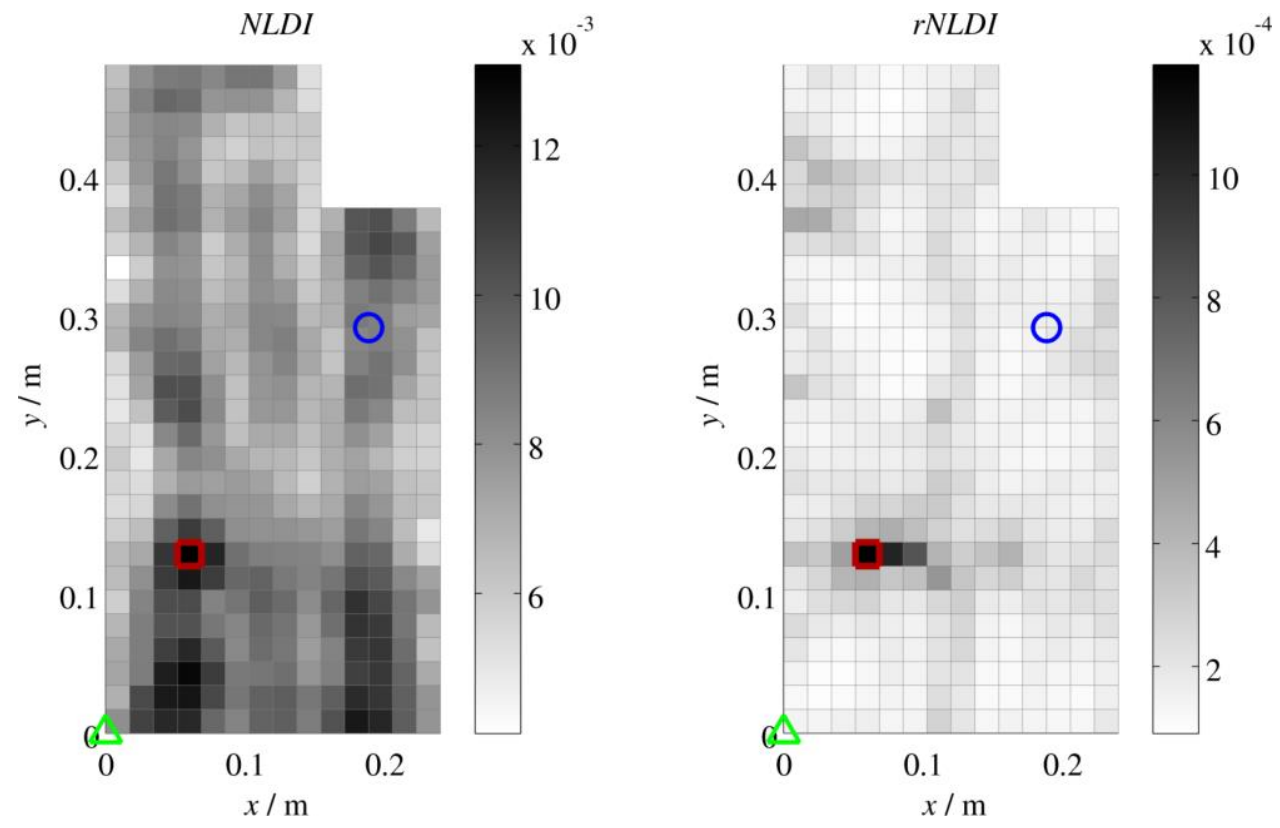

Fig. 10 Linear and reciprocal damage index for asymmetric flat geometry.

Damaged elements (degradation $\hat{r}=12.5 \%$ ) are highlighted red.

Excitation at $(0,0)$ (green). Nonlinear measure $\lambda$ is taken at $(0.19 \mathrm{~m}, 0.3 \mathrm{~m})$ (blue) 


\section{CONCLUSIONS AND FUTURE WORK}

The results presented here are showing that combining the linear modal description of a structure and slight nonlinearities triggered by resonant excitation is a suitable approach for damage localization. By simulating an experimental set up it is found that the two suggested formulations of a spatial nonlinear damage index are able to reliably identify a moderately large defective area. Especially to be emphasized is the fact that the nonlinear measure, which is the only value associated with the damage, can be determined at any structural point.

In the present paper the nonlinear damage indexes were processed in a very straightforward way - all first 42 bending modes were taken in account and the nonlinear measure is estimated as a sum of the first four higher harmonic amplitudes. In order to improve the validity of the damage localization more complex definitions, e.g., an appropriate selection of modes and/or discrimination between even and odd higher harmonics, might be adapted in future. For "real world" applications of this approach the assumed correlation between the modal shape and the nonlinear measure needs to be proven first. Preliminary experimental tests on a plate made of glass fiber-reinforced plastic show very promising results.

\section{REFERENCES}

1. Cai, J., Qiu, L., Yuan, S., Liang, D., Shi, L., Liu, P.P., Liang, D., 2012, Structural Health Monitoring for Composite Materials, Composites and Their Application, INTECH.

2. Balageas, D., Fritzen, C., Güemes, A. (Eds.), 2006, Structural Health Monitoring, ISTE, London, Newport Beach.

3. Masters, J. (Ed.), 1992, Damage detection in composite materials, 1128, American Society for Testing and Materials, Philadelphia.

4. Carden, E.P., Fanning, P., 2004, Vibration Based Condition Monitoring: A Review, Structural Health Monitoring, 3(4), pp. 355-377.

5. Doebling, S.W., Farrar, C.R., Prime, M.B., 1998, A Summary Review of Vibration-Based Damage Identification Methods, Identification Methods, Shock and Vibration Digest, 30(2), pp. 91-105.

6. Kaiser, S., Melcher, J., Breitbach, E., Sachau, D., 1999, Structural dynamic health monitoring of adaptive CFRP structures, Proc. SPIE 3674, Smart Structures and Materials 1999: Industrial and Commercial Applications of Smart Structures Technologies, 51.

7. Zou, Y., Tong, L., Steven, G., 2000, Vibration-Based Model-Dependent Damage (Delamination) Identification and Health Monitoring for Composite Structures - A Review, Journal of Sound and Vibration, 230(2), pp. 357-378.

8. Montalvão, D., Maia, N., Ribeiro, A., 2006, A Review of Vibration-Based Structural Health Monitoring with Special Emphasis on Composite Materials, Shock and Vibration Digest, 38(4), pp. 295-326.

9. Sohn, H., 2004, A Review of Structural Health Monitoring Literature: 1996-2001, Technical report, Los Alamos National Laboratory.

10. Salawu, O., 1997, Detection of structural damage through changes in frequency: a review, Engineering Structures, 19(9), pp. 718-723.

11. Philips Adewuyi, A., Wu, Z., Kammrujaman Serker, N., 2009, Assessment of Vibration-Based Damage Identification Methods using Displacement and Distributed Strain Measurements, Structural Health Monitoring, 8(6), pp. 443-461.

12. Montalvão, D., Ribeiro, A., Duarte-Silva, J., 2009, A Method for the Localization of Damage in a CFRP Plate Using Damping, Mechanical Systems and Signal Processing, 23(6), pp. 1846-1854.

13. Chattopadhyay, A., Kim, H., Ghoshal, A., 2004, Non-linear vibration analysis of smart composite structures with discrete delamination using a refined layerwise theory, Journal of Sound and Vibration, 273(1-2), pp. 387-407. 
14. Farrar, C.R., Worden, K., Todd, M.D., Park, G., Nichols, J., Adams, D.E., Bement, M.T., Farinholt, K., 2007, Nonlinear System Identification for Damage Detection, Technical report, LA-14353, Los Alamos National Laboratory.

15. Prime, M.B., Shevitz, D.W., 1996, Linear and Nonlinear Methods for Detecting Cracks in Beams, 14th International Modal Analysis Conference, IMAC XIV, Dearborn

16. Della, C.N., Shu, D., 2007, Vibration of delaminated composite laminates: A review, Applied Mechanics Reviews, 60(1), pp. 1-20.

17. Lestari, W., Hanagud, S., 1999, Health monitoring of structures: multiple delamination dynamics in composite beams, Proceedings of the 40th AIAA/ASME/ASCE/AHS/ASC structures, structural dynamics and materials conference and adaptive structures forum, St. Louis, MO.

18. Shen, M.H., Grady, J., 1992, Free vibrations of delaminated beams, AIAA journal, 30(5), pp. 1361-1370.

19. Lu, X., Lestari, W., Hanagud, S., 2001, Nonlinear vibrations of a delaminated beam, Journal of Vibration and Control, 7(6), pp. 803-831.

20. Ostachowicz W., Zak A., 2004, Vibration of a laminated beam with a delamination including contact effects, Shock and Vibration, 11(3), pp. 157-171.

21. Ostachowicz, W., Dulieu-Barton, J., Holford, K., Krawczuk, M., Zak, A., 2005, Key Engineering Materials Damage Assessment of Structures VI, Key Engineering Materials - Damage Assessment of Structures VI, 293 294(607), pp. 607-616.

22. Müller, I., 2007, Clapping in delaminated sandwich-beams due to forced oscillations, Computational Mechanics, 39(2), pp. 113-126.

23. Saravanos, D., Hopkins, D., 1996, Effects of delaminations on the damped dynamic characteristics of composite laminates: analysis and experiments, Journal of Sound and Vibration, 192(5), pp. 977-993.

24. Jenal, R., Staszewski, W., Klepka, A., Uhl, T., 2010, Structural Damage Detection Using Laser Vibrometers, $2^{\text {nd }}$ International Symposium on NDT in Aerospace 2010, Hamburg, Germany.

25. Campbell, F., 2010, Structural Composite Materials, ASM International.

26. Figueiras, J., 1983, Ultimate load analysis of anisotropic and reinforced concrete plates and shells, Ph.D. thesis, University College of Swansea.

27. Nguyen-Thanh, N., Rabczuk, T., Nguyen-Xuan, H., Bordas, S.P., 2008, A smoothed finite element method for shell analysis, Computer Methods in Applied Mechanics and Engineering, 198(2), pp. 165-177.

28. Klepka, A., 2013, Advanced Structural Damage Detection: From Theory to Engineering Applications, John Wiley \& Sons, Ltd, chapter 4. Nonlinear Acoustics, pp. 73-107.

29. Moussatov, A., Gusev, V., Castagnède, B., 2003, Self-Induced Hysteresis for Nonlinear Acoustic Waves in Cracked Material, Phys. Rev. Lett., 90.

30. Friswell, M.I., Penny, J.E., 2002, Crack modeling for structural health monitoring, Structural Health Monitoring, 1(2), pp. 139-148.

31. Courtney, C., Neild, S., Wilcox, P., Drinkwater, B., 2010, Application of the bispectrum for detection of small nonlinearities excited sinusoidally, Journal of Sound and Vibration, 329(20), pp. 4279-4293.

32. Klepka, A., Straczkiewicz, M., Pieczonka, L., Staszewski, W.J., Gelman, L., Aymerich, F., Uhl, T., 2015, Triple correlation for detection of damage related nonlinearities in composite structures, Nonlinear Dynamics, 81(1), pp. pp 453-468.

33. Hughes, T.J., 1983, Analysis of transient algorithms with particular reference to stability behavior, in Belytschko, T., Hughes, T.J., (Eds.), Computational Methods for Transient Analysis, Elsevier Science Publishers, pp. 67-155.

34. Heinzel, G., Rüdiger, A., Schilling, R., 2002, Spectrum and spectral density estimation by the Discrete Fourier transform (DFT), including a comprehensive list of window functions and some new at-top windows, Technical report, Max-Planck-Institut für Gravitationsphysik (Albert-Einstein-Institut). 